\title{
LA EVALUACIÓN EN EL DISEÑO ALINEADO DE CURSOS: LA EXPERIENCIA DE UN CURSO INTEGRADOR*
}

\author{
María Fernanda Aldana Vargas** \\ David Schnarch González ${ }^{* * *}$
}

Resumen: Este artículo ilustra un ejercicio de análisis y rediseño de un sistema de evaluación de un curso integrador desarrollado en la Facultad de Administración de la Universidad de los Andes cuya metodología se basa en el "aprender haciendo". Se describe el proceso llevado a cabo por los autores y se muestra cómo, a partir del proceso de evaluación, se motivó el alineamiento en objetivos de aprendizaje, actividades, desempeños y criterios de evaluación. De esta forma, la experiencia ejemplifica tres aspectos centrales que contribuyen a una evaluación de calidad: evaluación alineada con el currículo, evaluación para el aprendizaje y evaluación auténtica.

Palabras-clave: Consultoría, alineación curricular, aprender haciendo.

Resumo: Este artigo ilustra um exercício de análise e redesenho de um sistema de avaliação de um curso integrador desenvolvido na Faculdade de Administração da Universidade dos Andes cuja metodologia se baseia no "aprender fazendo". Descreve-se o processo levado a cabo pelos autores e mostra-se como, a partir do processo de avaliação, se motivou o alinhamento em objetivos de aprendizagem, atividades, desempenhos e critérios de 
avaliação. Desta forma, a experiência exemplifica três aspectos centrais que contribuem a uma avaliação de qualidade: avaliação alinhada com o currículo, avaliação para a aprendizagem e avaliação autêntica.

Palavras-chaves: Consultoria. Alinhamento curricular. Aprender fazendo.

Abstract: This article illustrates an analysis exercise and redraw a system of evaluation of a course integrator developed at course of Administration of the Andes University whose methodology bases on "learning doing". The process is described taken to cable by the authors and it is shown as, starting from the evaluation process, the alignment was motivated in learning objectives, activities, actings and evaluation criteria. This way, the experience exemplifies three central aspects that contribute it a quality evaluation: aligned evaluation with the curriculum, evaluation for the learning and authentic evaluation.

Keywords: Consultancy. Alignment curriculum. To learn doing. 


\section{Introducción}

Evaluar el aprendizaje es un reto que todo docente enfrenta. Aunque en muchos casos se tiene clara la intencionalidad detrás de las actividades de clase, no necesariamente éstas se encuentran alineadas con los objetivos de aprendizaje o con los mecanismos de evaluación utilizados. Es común que exista una desconexión entre lo que el profesor evalúa y lo que quiere desarrollar en el estudiante. Este trabajo precisamente aborda esa problemática en un curso basado en el "aprender haciendo" donde existen dos elementos centrales: una aproximación hacia la comprensión de cómo evaluar un curso centrado en la práctica y la relación entre la evaluación del aprendizaje y de la práctica como tal. En ese sentido, la pregunta que se intenta resolver es: ¿Cómo orientar la evaluación de un curso centrado en la práctica que dé cuenta del aprendizaje y de la práctica misma?

Para el cumplimiento de este propósito el documento se estructura de la siguiente manera. En la primera parte se describe el curso, el entorno en el cual se enmarca la necesidad y los antecedentes. A continuación se esquematiza el análisis y el rediseño del sistema de evaluación. Los últimos apartados presentan la reflexión acerca del proceso y se presentan las conclusiones que aluden a la manera en la que el desarrollo del ejercicio ha permitido responder a conocimiento sobre evaluación.

\section{Descripción del Curso Casos y Proyectos en Administración ${ }^{1}$}

"Casos y Proyectos en Administración" es un curso de últimos semestres del programa de Pregrado en Administración que ofrece 
al estudiante un espacio para desarrollar una práctica que integre los conocimientos adquiridos durante el programa. Desarrollada en un entorno real, dicha práctica aporta al desarrollo de competencias profesionales en administración y al entendimiento del enfoque crítico en el aprendizaje. Específicamente, se pretende fortalecer la capacidad de conceptualización para analizar problemas complejos con el fin de hacerlos gestionables.

En este sentido, el curso está diseñado en el marco de una firma consultora - ConsultAndes - donde los estudiantes se ven involucrados en el desarrollo de una metodología de consultoría en Administración. Esta metodología está orientada a la investigación, al trabajo en equipo, a la visión integral de las organizaciones, a la toma de decisiones y a la comunicación para dar solución a una necesidad real de un cliente. Adicionalmente, los estudiantes se familiarizan con la consultoría como herramienta de administración para el fortalecimiento de las organizaciones o como campo de acción central para su desarrollo y desempeño profesional.

Los proyectos se realizan en equipos de tres a cuatro estudiantes bajo la supervisión de un profesor y se discuten las metodologías de trabajo que incluyen prácticas de consultoría, gestión de proyectos y asesorías especializadas sobre temáticas particulares.

Las áreas de trabajo del programa incluyen disciplinas de la Administración como mercadeo, finanzas, gestión, estrategia y operaciones \& logística. Algunos ejemplos de proyectos desarrollados bajo el programa ConsultAndes son: estudios de mercado, estudios de pre-factibilidad de proyectos, planteamiento de nuevas estrategias de negocio, desarrollo de nuevos productos, análisis y estructuración de sistemas de costos, planeación operacional, implementación de nuevas tecnologías o sistemas, entre otros. 
A agosto de 2011, ConsultAndes ha desarrollado 240 proyectos de consultoría (25 por semestre, aproximadamente) con un total de 176 organizaciones en diferentes sectores de la economía colombiana, de las cuales $74.4 \%$ son PYMES, $14.2 \%$ ONG y $10.8 \%$ grandes empresas. Se ha contado, además, con la participación de 1.089 estudiantes de pregrado desde que inició el curso en 2006 hasta el primer semestre de 2011.

El curso está compuesto por un equipo de cinco profesores, cinco monitores y un coordinador, quienes acompañan y asesoran a un grupo de 100 estudiantes por semestre, en promedio. Cada profesor se encarga, entonces de dirigir 5 proyectos de consultoría (cerca de 25 estudiantes).

Adicionalmente, ConsultAndes cuenta con "Comités Directivos" de seguimiento a cada proyecto donde participan el representante del cliente, el equipo consultor, colegas del curso, el monitor y el profesor. Estos comités son espacios de retroalimentación y orientación de avances que se llevan a cabo tres veces al semestre.

En concordancia con lo anterior, los objetivos de aprendizaje del curso son:

- Visión integral de las organizaciones: Los estudiantes tendrán una visión integral de las organizaciones a partir de un sólido conocimiento de las áreas funcionales de la administración.

- Toma de decisiones: Los estudiantes identificarán oportunidades y propondrán soluciones innovativas a situaciones de las organizaciones, así como proyectos emprendedores.

- Comunicación: Los estudiantes se comunicarán en forma escrita y oral de manera clara, coherente y efectiva.

- Trabajo en equipo: Los estudiantes trabajarán con otros de forma comprometida, cooperativa y coordinada. 
La metodología del programa parte de los principios básicos del "aprender haciendo" por medio de servicios. Esta aproximación a situaciones de la vida real genera los siguientes elementos:

- Permite aplicar conceptos de administración a problemas reales (GODFREY et al., 2005).

- Genera un espacio para fortalecer sensibilidades, comportamientos éticos y participación ciudadana (SALIMBENE et al., 2005).

- Motiva a contribuir a la comunidad en una forma constructiva mientras se aprenden conceptos académicos (VEGA, 2007).

- Genera retroalimentación personal y directa sobre la aplicación y limitaciones de conceptos teóricos en un ambiente de práctica imprevisible (KENWORTHY-U'REN, 2005).

De esta manera el aprendizaje durante el programa se manifiesta mediante un proceso cíclico en donde estudiantes adquieren experiencias particulares a través de actividades concretas y las conviertan en aprendizajes y conceptualizaciones por medio de reflexiones y retroalimentación académica (KOLB, 2005). A través de la metodología "aprender haciendo", el estudiante construye conocimiento profesional aplicado, fortalece sus capacidades de razonamiento y solución de problemas, desarrolla estrategias de auto-aprendizaje, se motiva para el aprendizaje y avanza en su proceso de convertirse en un colaborador efectivo (SHERWOOD, 2004).

La siguiente tabla muestra las diferentes características de los involucrados en el proceso pedagógico y la dinámica académica utilizada en "el aprender haciendo": 
Tabla 1 - Características del "aprender haciendo"

\begin{tabular}{|c|l|}
\hline Involucrado/elemento & \multicolumn{1}{c|}{ Característica } \\
\hline Rol del estudiante & $\begin{array}{l}\text { Liderazgo sobre su proceso de aprendizaje. } \\
\text { Construcción de conocimiento a partir de expe- } \\
\text { riencias personalizadas. } \\
\text { Integración de conocimientos y competencias }\end{array}$ \\
\hline Rol del profesor & $\begin{array}{l}\text { Coordina el proceso de aprendizaje, más no lo } \\
\text { lidera. } \\
\text { Facilitador, acompañamiento personalizado. } \\
\text { Guía, orientador. } \\
\text { Retroalimenta. } \\
\text { Provee información. }\end{array}$ \\
\hline Compañeros de equipo & $\begin{array}{l}\text { Mecanismo y fuente de aprendizaje. } \\
\text { Fuente de retroalimentación y reflexión. }\end{array}$ \\
\hline Cliente & $\begin{array}{l}\text { Mecanismo y fuente de aprendizaje. } \\
\text { Fuente de retroalimentación y reflexión. }\end{array}$ \\
\hline Dinámica académica & $\begin{array}{l}\text { Proceso cíclico y acumulativo de aprendizajes. } \\
\text { Errores y conflictos se valoran como proceso de } \\
\text { aprendizaje. } \\
\text { Proceso real (no simulación). } \\
\text { Proceso de evaluación integral (profesor, cliente, } \\
\text { compañeros). }\end{array}$ \\
\hline
\end{tabular}

\section{Antecedentes del proceso}

En 2001 la Facultad de Administración de la Universidad de los Andes inició un proceso de acreditación internacional ${ }^{2}$ que motivó cambios en su estructura y aproximación a la docencia. Fue así como en 2005 definió una serie de 'competencias' centrales que buscarían desarrollarse en el pregrado y se impulsó el uso de metodologías centradas en el estudiante. Dos años más tarde se reformó el currículo de pregrado, se definieron siete objetivos de aprendizaje del programa y se establecieron objetivos de aprendizaje para cada curso. 
La reforma establecía que cada curso debía aportar a determinado(s) objetivo(s) del pregrado. En la misma línea, se definieron cursos "integradores" 3 donde se medirían varios de estos objetivos y se daría un reporte sobre el estado de los mismos en el marco general del programa. Casos y proyectos en Administración fue seleccionado como uno de esos “integradores” en 2008, lo cual implicó un reto importante para el equipo de profesores del curso: ¿Cómo medir el desarrollo de esos objetivos de aprendizaje? Realmente no se tenían herramientas para hacerlo. Hasta el momento la evaluación del curso se hacía en el marco de lo que se entendía como un "proyecto de consultoría exitoso", no en un "proceso de aprendizaje exitoso". Aunque estas dos aproximaciones están íntimamente relacionadas, con las herramientas de evaluación no era posible presentar evidencia sobre esta segunda aproximación.

\section{Análisis y rediseño del sistema de evaluación}

Producto de una reflexión acerca de los logros alcanzados y sobre la dinámica propia de la metodología el grupo de profesores ha realizado ajustes al diseño pedagógico del curso semestre a semestre desde el inicio del mismo (2006). Fue así como, en el primer semestre del 2011, se inició el rediseño de la evaluación del aprendizaje de manera que estuviera en correspondencia con la particularidad de la metodología y que favoreciera la definición de lineamientos compartidos por el equipo de profesores. Esta tarea se desarrolló con el acompañamiento de un equipo pedagógico del CIFE 4 . 
El proceso para el rediseño contempló cuatro momentos: diagnóstico de necesidades asociadas a la evaluación del aprendizaje; análisis de los componentes del curso y, específicamente, de la práctica de la consultoría; diseño alineado de la evaluación; implementación y seguimiento a la misma. El presente artículo recoge los tres momentos iniciales, centrados en el diseño (la implementación está en curso).

Diagnóstico de necesidades asociadas a la evaluación del aprendizaje

Este momento se centró en la identificación de las condiciones de la evaluación y definición de líneas de trabajo. Se basó en el balance realizado por los profesores $y$, a partir de esto, se reconocieron las siguientes necesidades:

- Requisitos de la evaluación para un curso integrador: El curso plantea el desarrollo de competencias y de objetivos de aprendizaje alineados con los objetivos del programa de pregrado. El carácter de "curso integrador" exigió especial claridad sobre la evaluación de estos propósitos y su mutua correspondencia.

- Correspondencia entre los objetivos de aprendizaje y los objetivos de la consultoría: Puesto que el curso responde a dos propósitos generales (el desarrollo de aprendizaje sobre la consultoría y el desarrollo de una asesoría para un cliente en particular) se hizo necesario definir y clarificar la correspondencia entre unos y otros. Fue posible encontrar casos en que los estudiantes lograron aprendizajes significativos aún en situaciones de consultorías no exitosas (determinadas por condiciones del entorno empresarial 
abordado). Este aspecto requirió definir el concepto de "consultoría exitosa”, el alcance esperado para la misma y la evaluación del aprendizaje, independientemente del éxito de la misma.

- Participación de los distintos actores en la evaluación: La metodología basada en el "aprender haciendo" involucra diversos actores: profesor, monitor, cliente y el estudiante mismo. En correspondencia, la evaluación integra procesos de autoevaluación, heteroevaluación y coevaluación, que requieren ser basados en una visión y práctica compartida. Se hizo prioritario especificar la forma de participación de los distintos actores involucrados, ya sea para el desarrollo de la consultoría o para promover el aprendizaje de los estudiantes, así como generar acuerdos sobre la práctica misma.

Coincidiendo con Biggs (1999), quien señala como principio de una evaluación para un aprendizaje de calidad, que las tareas de evaluación comprendan una auténtica representación de los objetivos del curso e identifica como característica de una buena evaluación, el asegurar su alineación con el currículo. Se decidió abordar aquella enunciada en segundo término: correspondencia entre objetivos de aprendizaje y objetivos de la consultoría. Para ello se consideró la alineación de la actividad de consultoría con los objetivos del curso y la evaluación de la misma.

Asimismo, esta necesidad permitía dar respuesta a una pregunta reconocida como esencial para el curso: 


\section{¿Cómo orientar la evaluación de un curso centrado en la práctica, que dé cuenta del aprendizaje y de la práctica misma?}

Para construir una respuesta a esta pregunta se llevó a cabo una exploración de la evaluación implementada hasta el momento, basada en la revisión documental y en la experiencia de docentes y monitores. Este análisis se abordó desde una perspectiva de alineación de los componentes del curso, así, a medida que se reconocían los desarrollos alcanzados, se consideraba el sentido, la pertinencia y orientación de los mismos.

Análisis de los componentes del curso y de la práctica de la consultoría

Este momento de análisis estuvo orientado por el principio de alineación curricular, es decir, en la importancia de alinear los objetivos, la enseñanza y la evaluación en una experiencia de aprendizaje Palomba \& Banta (2001). Para dar inicio al análisis, se estudiaron dos componentes centrales como son la consultoría y los objetivos de aprendizaje. Esto permitió explicitar y clarificar aquello que debían aprender los estudiantes. Al tener la definición y comprensión de cada componente se pasó a identificar puntos de encuentro o de diferenciación entre los mismos.

Se entendió la consultoría como aprendizaje práctico, es decir, como espacio de experiencia real en el que se desempeñan los estudiantes marcado por la complejidad propia de un contexto particular, que a la vez que le plantea al estudiante retos y desafíos, le ofrece la posibilidades de aplicación de sus comprensiones. Está 
asociado a un conocimiento funcional, en el que se ve involucrada la experiencia del aprendiz y su comprensión sobre la misma. En términos de Biggs (1999, p. 64) sería aquel que "[...] requiere un sólido fundamento de conocimientos declarativos [...] pero también implica saber cómo hacer las cosas, cómo desarrollar procedimientos o aplicar destrezas y cuándo hacer estas cosas y por qué.”

$\mathrm{Al}$ estar la práctica enmarcada en una firma consultora (ConsultAndes), toma particular relevancia el logro de un desempeño "exitoso" en el desarrollo de la misma, así, tanto los estudiantes como los profesores asesores tienen el apremio de alcanzar una consultoría exitosa en la que es indispensable generar un valor al cliente en la prestación del servicio. Con el propósito de hacer explícita esta exigencia y sus implicaciones, el grupo de profesores desarrolló la descripción de consultoría exitosa que se presenta en la siguiente tabla.

Tabla 2 - Matriz de Consultoría exitosa ${ }^{5}$

\begin{tabular}{|c|l|}
\hline Factor & \multicolumn{1}{|c|}{ Descripción } \\
\hline Creación de valor & $\begin{array}{l}\text { Las recomendaciones del equipo consultor efectiva- } \\
\text { mente identificaron una creación de valor relevante al } \\
\text { cliente }\end{array}$ \\
\hline Relacionamiento & $\begin{array}{l}\text { Las interacciones con el cliente lograron un servicio di- } \\
\text { ferenciador y relaciones de alto impacto }\end{array}$ \\
\hline Implementación & $\begin{array}{l}\text { Como resultado del proyecto, el cliente aceptó las reco- } \\
\text { mendaciones del equipo consultor y las está ejecutando }\end{array}$ \\
\hline Costo-Beneficio & $\begin{array}{l}\text { El tiempo dedicado por parte del cliente al proyecto jus- } \\
\text { tificó la vinculación de la empresa al programa con base } \\
\text { en las recomendaciones recibidas y la creación de valor } \\
\text { identificada }\end{array}$ \\
\hline Confianza & $\begin{array}{l}\text { Las habilidades que exhibió el equipo consultor forta- } \\
\text { lecieron la confianza depositada en éste durante todo el } \\
\text { proyecto }\end{array}$ \\
\hline
\end{tabular}




\begin{tabular}{|c|l|}
\hline Fidelidad & $\begin{array}{l}\text { El cliente confía en ConsultAndes para volverlo a con- } \\
\text { tratar para futuros proyectos }\end{array}$ \\
\hline Fundamentos & $\begin{array}{l}\text { El grupo consultor evidenció una sólida formación y } \\
\text { aplicación de su formación académica a través de mar- } \\
\text { cos metodológicos apropiados }\end{array}$ \\
\hline Creatividad & $\begin{array}{l}\text { El grupo consultor aportó nuevas ideas que permitieron } \\
\text { crear espacios de creatividad para lograr una diferenciaci- } \\
\text { ón del cliente clara frente a sus principales competidores }\end{array}$ \\
\hline
\end{tabular}

Los criterios elaborados por los profesores sobre la consultoría exitosa evidencian los desempeños que le demanda el contexto de un entorno empresarial al estudiante, así como la complejidad que representa aproximarse a los intereses de múltiples actores (la organización, el cliente y grupos de interesados).

Para abordar el segundo componente - objetivos de aprendizaje - fue necesario considerar su relación con cada momento de la consultoría, pues existe el riesgo de focalizarse en cumplir con las demandas de ésta y pensar que, al satisfacerlas, los estudiantes logran los objetivos de aprendizaje. Es importante detenerse en este aspecto para relatar una de las situaciones que surge en un curso centrado en el aprender haciendo y en experiencias que integran el entorno académico con los entornos sociales: La presión de responder a las necesidades, condiciones y dinámicas de un contexto organizacional en particular, representa un reto constante para el grupo de estudiantes y para el profesor asesor, haciendo que en ocasiones, los objetivos de aprendizaje se asuman como implícitos al proceso.

Con el propósito de atender esta situación, se especificaron los momentos centrales de la consultoría, ahora vista como aprendizaje práctico, como desempeño del estudiante que da cuenta de los aprendizajes esperados. De esta manera, se centró la atención en los aprendizajes que se espera desarrolle el estudiante a lo largo de esta práctica como se muestra en la siguiente tabla. 
Tabla 3 - Consultoría como desempeño del estudiante

\begin{tabular}{|c|c|}
\hline $\begin{array}{l}\text { Consultoría como } \\
\text { desempeño }\end{array}$ & $\begin{array}{l}\text { Conocimientos asociados al desempeño } \\
\text { (conceptos, procedimientos, actitudes) }\end{array}$ \\
\hline $\begin{array}{l}\text { Primer contacto y venta de } \\
\text { consultoría: Diagnóstico de la } \\
\text { necesidad y elaboración de la } \\
\text { propuesta }\end{array}$ & $\begin{array}{l}\text { Conceptuales: } \\
\text { - Consultoría como herramienta de admi- } \\
\text { nistración. } \\
\text { Procedimentales: } \\
\text { - Reconocimiento de la necesidad del cliente. } \\
\text { - Conceptualización del problema. } \\
\text { - Comunicación de la propuesta. } \\
\text { Actitudinales: } \\
\text { - Trabajo en Equipo. }\end{array}$ \\
\hline $\begin{array}{l}\text { Análisis integral de la nece- } \\
\text { sidad: } \\
\text { Compresión sistémica del pro- } \\
\text { blema }\end{array}$ & $\begin{array}{l}\text { Procedimentales: } \\
\text { - Compresión amplia de la necesidad del } \\
\text { cliente. } \\
\text { Conceptuales y procedimentales: } \\
\text { - Diseño de las actividades sustentadas en el } \\
\text { marco conceptual seleccionado. }\end{array}$ \\
\hline $\begin{array}{l}\text { Investigación de alternativas } \\
\text { o escenarios }\end{array}$ & $\begin{array}{l}\text { Conceptuales: } \\
\text { - Aplicación de herramientas metodológi- } \\
\text { cas para la identificación de alternativas. } \\
\text { Procedimentales: } \\
\text { - Indagación y análisis del valor agrega- } \\
\text { do de las alternativas frente a la necesidad } \\
\text { planteada por el cliente (real y sentida). } \\
\text { - Generación de propuestas innovadoras. } \\
\text { - Identificación de la relación entre las alter- } \\
\text { nativas y los referentes conceptuales. } \\
\text { - Comunicación del informe de avance. } \\
\text { Actitudinales: } \\
\text { - Trabajo en equipo. }\end{array}$ \\
\hline $\begin{array}{l}\text { Planteamiento y venta de re- } \\
\text { sultados: } \\
\text { Lograr la apropiación de los } \\
\text { resultados por parte del cliente. }\end{array}$ & $\begin{array}{l}\text { Procedimentales: } \\
\text { - Análisis del valor agregado de los resul- } \\
\text { tados frente a los objetivos y necesidad del } \\
\text { cliente. } \\
\text { - Comunicación final del proyecto. } \\
\text { Actitudinales: } \\
\text { - Relación con el cliente. } \\
\text { - Trabajo en Equipo. }\end{array}$ \\
\hline
\end{tabular}




\begin{tabular}{|l|l|}
\hline $\begin{array}{l}\text { Reflexión sobre los aprendi- } \\
\text { zajes y lecciones obtenidas en } \\
\text { el curso }\end{array}$ & - Conceptuales: \\
& ciones aprendidas sobre la consultoría \\
& como herramienta de administración. \\
& - Análisis de los elementos de la consultoría \\
& aplicados en diferentes contextos. \\
& Procedimentales: \\
& - Comunicación. \\
\hline
\end{tabular}

La construcción de la consultoría como desempeño condujo a una reflexión detenida sobre los momentos que la configuran y sobre la relevancia formativa de los mismos. Hizo visibles intencionalidades de la práctica educativa que no eran reconocidas como tal y, por tanto, no eran necesariamente compartidas por el grupo de docentes, aunque podían llegar a ser aspectos relevantes a la hora de evaluar. Por otra parte, este ejercicio llevó a reconocer los procesos que podrían asociarse a la enseñanza, en este caso a reconocer el objetivo de ciertas actividades orientadas a potenciar la consultoría.

La construcción de la consultoría como aprendizaje permitió avanzar hacia el diseño de su evaluación. El desempeño fue analizado en relación con los objetivos de aprendizaje y fue tomado como eje alrededor del cual se especificaron estrategias de enseñanza, conocimientos y criterios para su evaluación.

\section{Diseño alineado de la evaluación}

Las especificaciones construidas en el primer y segundo momento permitieron llegar con los elementos claros para un diseño alineado de la evaluación. Ésta se enfocó en la revisión de los objetivos, actividades y evaluación, así como en la relación entre los 
mismos. A continuación se presenta un esquema que permitió esta mirada relacional y en las gráficas siguientes, algunos de los tipos de resultados de este proceso.

Tabla 4 - Esquema para la alineación de componentes ${ }^{6}$

\section{Objetivos del curso}

Lo que se espera que el estudiante comprenda y sea capaz de hacer como resultado del curso.

\begin{tabular}{|c|c|c|c|}
\hline Actividad & Desempeño & Conocimiento & Criterio \\
\hline $\begin{array}{l}\text { Son las unidades de ac- } \\
\text { tuación en el proceso de } \\
\text { enseñanza-aprendizaje. } \\
\text { Se constituyen de for- } \\
\text { ma integrada, esto es, } \\
\text { en ellas están presentes } \\
\text { los objetivos formativos } \\
\text { (son los que les dan sen- } \\
\text { tido), como la actuación } \\
\text { de los profesores (quie- } \\
\text { nes definen los requeri- } \\
\text { mientos) y de los estu- } \\
\text { diantes (quienes han de } \\
\text { llevar a cabo la actividad } \\
\text { diseñada). }\end{array}$ & $\begin{array}{l}\text { Acciones de los alum- } \\
\text { nos que hacen obser- } \\
\text { vable su aprendizaje. A } \\
\text { partir de varios desem- } \\
\text { peños se puede inferir } \\
\text { el logro del objetivo } \\
\text { o el desarrollo de la } \\
\text { competencia. Tienen } \\
\text { un carácter de autenti- } \\
\text { cidad en la medida en } \\
\text { que se desarrollan en } \\
\text { un contexto real. }\end{array}$ & $\begin{array}{l}\text { Aquellos que se evi- } \\
\text { dencian en el desem- } \\
\text { peño: conceptos, teor- } \\
\text { ías, modelos, normas, } \\
\text { procedimientos. }\end{array}$ & $\begin{array}{l}\text { Especifica niveles de } \\
\text { desempeño. Permite } \\
\text { estimar cualidades } \\
\text { propias frente a los } \\
\text { objetivos esperados. }\end{array}$ \\
\hline
\end{tabular}

La mirada integrada de los objetivos y los momentos de la consultoría ofrecieron claridad sobre los conocimientos asociados e igualmente sobre los criterios que guiarían una evaluación contextualizada, la cual va más allá de un conocimiento exclusivamente declarativo ${ }^{7}$ extendiéndose a un conocimiento de tipo relacional y funcional ${ }^{8}$, en un ambiente de aprendizaje que ofrece posibilidades de aplicación de lo aprendido en un contexto profesional.

El siguiente diagrama muestra la relación entre los momentos de la consultoría y los conocimientos (asociados a los objetivos), que se promueven en cada uno: 
Gráfica 1 - Conocimientos alineados a los momentos de desempeño de consultoría

\section{Consultoría como desempeño}

Primer contacto y venta de consultoría:

Diagnóstico de la necesidad y

elaboración de la propuesta

Análisis integral de la necesidad:

2. Compresión sistémica del problema

(B) Investigación de alternativas o escenarios

Planteamiento y venta de resultados:

Lograr la apropiación de los resultados

por parte del cliente.

Reflexión sobre los aprendizajes

y lecciones obtenidas en el curso

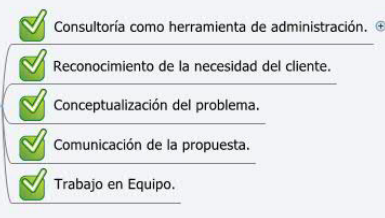

Trabajo en Equipo.

\section{A continuación se presenta la ruta para llegar a los criterios de} evaluación para cada momento de la consultoría:

Gráfica 2 - Criterios alineados a los momentos del desempeño de la consultoría

\section{$\oplus$}


Como se dijo anteriormente, este ejercicio permitió hacer explícito el propósito de la enseñanza y las actividades asociadas a la misma, es decir, el diseño del curso para promover el aprendizaje:

Gráfica 5 - Actividades alineadas a los momentos del desempeño de la consultoría

\section{Consultoría como desempeño}

Lectura individual

Primer contacto y venta de consultoría:

Diagnóstico de la necesidad y

elaboración de la propuesta

Análisis integral de la necesidad:

Compresión sistémica del problema

Investigación de alternativas o escenarios

Planteamiento y venta de resultados:

4. Lograr la apropiación de los resultados

por parte del cliente.

Reflexión sobre los aprendizajes

y lecciones obtenidas en el curso
Taller de preparación y

primer contacto con el cliente

Taller Proven Models

Asesorias de preparación de

propuestas y conceptualización de la necesidad del cliente

El ejercicio se extendió a la totalidad de las actividades de enseñanza y aprendizaje del curso. Una vez definida esta estructura de la evaluación, alineada con los componentes, se seleccionaron los instrumentos y las rúbricas para la valoración. Varios de ellos estuvieron asociados a las actividades de aprendizaje, siendo este un elemento más que muestra la alineación que orientaba el ejercicio.

\section{Reflexiones de profesor}

Es interesante observar cómo una necesidad puntual - centrada en la medición del cumplimiento de los objetivos de apren- 
dizaje - invitó a analizar la alineación de los diferentes elementos del curso y llevó a replantear la visión compartida de éste, sus componentes y su relevancia en el proceso de aprendizaje de los estudiantes.

El primer elemento que se destacó en este proceso se enmarcó en el análisis de las actividades centrales en el proceso de aprendizaje de los estudiantes. El programa del curso ya establecía una serie de desempeños. Sin embargo, algunos de ellos no se encontraban directamente relacionados con los conocimientos ni objetivos que el curso pretendía medir. Esta situación era percibida por los estudiantes como una "separación"9 entre su proyecto y las sesiones de clase. ¿A qué objetivo contribuye esta actividad? Cuando no encontrábamos respuesta, sabíamos que era necesario cambiar la naturaleza de la misma. Del mismo modo, en este proceso evidenciamos que existían objetivos de aprendizaje que no se abordaban directamente con desempeños específicos, lo cual llevó a vincular nuevas actividad de aprendizaje y sus respectivas formas de evaluación. De esta forma se implementaron dos nuevos talleres y un examen parcial.

En segundo lugar, el ejercicio de análisis permitió un diálogo entre profesores para homogenizar sus visiones del curso y los resultados esperados. Antes cada uno tenía una interpretación diferente de los objetivos (muchos de ellos concentrados únicamente en la satisfacción del cliente) y la retroalimentación que hacían no siempre estaba alineada. Poner de relieve el enfoque académico del curso unificando significados y criterios permitió tener una visión compartida del curso. Aunque no se pretende eliminar las diferencias en las apreciaciones y aproximaciones de cada profesor, ahora es más transparente para todos los integrantes del curso (profesores, estudiantes, monitores y clientes) cuáles son esos desempeños deseados y cómo éstos contribuyen a los objetivos de aprendizaje. 
Por último, la formulación del proceso de consultoría como desempeño invitó a dar mayor organicidad en el desarrollo de los proyectos. Ahora es más claro para cada profesor qué esperar en cada etapa del proyecto y hacia dónde dirigir a los equipos para que tengan un proceso de aprendizaje exitoso y una consultoría que genere valor al cliente. Esto último pone de relieve un elemento determinante en el éxito de este ejercicio: alinear los objetivos con actividades, conocimientos, desempeños y evaluación invitó a mejorar el proceso de consultoría y, esperamos, también de los resultados finales.

\section{Conclusiones}

El ejercicio realizado evidencia el potencial de la evaluación para lograr un diseño alineado de un ambiente de aprendizaje. Si bien el propósito de este ejercicio de diseño curricular siempre estuvo centrado en los retos de la evaluación, la construcción de alternativas - orientadas por la alineación - condujo a la revisión de todos los componentes del curso y especialmente a la reflexión sobre el quehacer de los profesores. El hacer explícitos propósitos, concepciones y acciones en torno a la enseñanza potenció la intencionalidad educativa de la práctica docente y la forma de vincular en el ejercicio a los diferentes actores: estudiante, profesor asesor, monitores y clientes.

La experiencia lograda hasta el momento permite plantear posibles respuestas a la pregunta enunciada en el primer momento de trabajo: ¿Cómo orientar la evaluación de un curso centrado en la práctica, que dé cuenta del aprendizaje y de la práctica misma? 
- Reconocer la naturaleza de los dos componentes: el aprendizaje y la práctica como tal, acompañado de una reflexión continua sobre la intencionalidad educativa de cada componente.

- Valorar el componente práctico desde su aporte a los aprendizajes propuestos y potenciar su desarrollo desde dicha intencionalidad. - Definir la alineación entre los elementos que configuran el ambiente, - actividades de enseñanza, evaluación, objetivos de aprendizaje - en donde éstos últimos se constituyen en el orientador del proceso.

- Sostener una visión sistémica en la que se reconoce la interdependencia de los componentes. Reconocer cómo la práctica orienta el aprendizaje y el aprendizaje orienta la práctica.

Por último, es necesario clarificar de qué manera este ejemplo desarrollado en el curso “Casos y Proyectos en Administración” permite responder a un conocimiento sobre la evaluación del aprendizaje. Para ello se toma un concepto utilizado por Biggs (1999) que ayuda a exponer uno de los aportes de esta experiencia. Este autor hace la distinción entre repercusión negativa y positiva de la evaluación. En la primera, no se encuentra una alineación entre actividades, objetivos y evaluación. El currículo real para el estudiante es lo evaluado y, en esa medida, se da un aprendizaje superficial, orientado en responder a la evaluación, "cuando la evaluación determina el aprendizaje y no el currículo oficial” (BIGGS, 1999, p. 177). Por otra parte, la repercusión positiva se da cuando la evaluación refleja los objetivos y cuando las actividades de enseñanza del profesor y las actividades de aprendizaje del estudiante se encaminan a estos mismos objetivos, es decir, todo gira en torno al aprendizaje.

Por otra parte, el diseño de la evaluación de este curso puede catalogarse como una evaluación auténtica o de la actuación, en 
la medida en que está claramente asociada a los objetivos y actividades de carácter práctico, entendido como aquel en el que se enseña "[...] a los estudiantes con el fin de que piensen, decidan y actúen en el mundo real de un modo más informado y eficaz: de manera ejecutiva" (WIGGINS, 1989; TORRANCE, 1994), citados por Biggs (1999). En este tipo de cursos, la evaluación requiere "una demostración activa del conocimiento en cuestión en contraste con hablar o escribir sobre el." (BIGGS, 1999, p. 189). Así mismo, puede ser definida como una evaluación contextualizada que está asociada a una práctica por medio de la cual se evalúa el conocimiento funcional (que a su vez articula el conocimiento declarativo y el procedimental), como es el caso de la evaluación de la consultoría en el curso estudiado. En "Casos y Proyectos en Administración" tanto las actividades, - consultoría -, como la evaluación, articulan el conocimiento funcional y declarativo.

Es necesario, ahora, medir y evaluar el impacto que está teniendo la aplicación de estos elementos en el curso. Dado que este semestre se están implementando las modificaciones al currículo y mecanismos de evaluación, resulta muy apresurado calificar este proceso como "exitoso". Sin embargo, las primeras aproximaciones evidencian que es una experiencia valiosa e interesante.

\section{Referencias}

BIGGS, J. Calidad del aprendizaje universitario. 3. ed. Madrid, España: Narcea S.A Ediciones, 1999.

GODFREY, P. C.; ILLES, L. M.; BERRY, G. R. Creating breadth in business education through service-learning. Academy of Management Learning \& Education, v. 4, p. 309-323, 2005. 
KENWORTHY-U'REN, A. Toward a scholarship of engagement: A dialogue between Andy Van de Ven and Edward Zlotkowski. Academy of Management Learning \& Education, v. 4, p. 272-277, 2005.

KOLB, A. Y.; KOLB, D. A. Learning styles and learning spaces: Enhancing experiential learning in higher education. Academy of Management Learning \& Education, v. 4, p. 193-212, 2005.

PALOMBA, C. A.; BANTA, T. W. Assessing student competence in accredited disciplines: pionnering approaches to assessment in higher education. 1. ed. Canada: Stylus Publishing, 2001.

ROBINSON, D.; LLOYD, A.; DEPAOLO, C. Service-Learning by Doing: How a Student-run Consulting Capstone Course. Journal of Management Education, v. 34, 2010.

SALIMBENE, F.; BUONO, A.; LAFRAGE, V.; NURICK, A. Servicelearning and management education: The Bentley experience. Academy of Management Learning \& Education, v. 4, p. 336-344, 2005.

SHERWOOD, A. L. Problem-based learning in management education: A framework for designing context. Journal of Management Education, v. 28, p. 536-557, 2004.

VEGA, G. Teaching business ethics through service-learning metaprojects. Journal of Management Education, v. 31, p. 647-678, 2007. 


\section{Notas}

* Trabajo presentado para el III Coloquio Internacional de la RIIED. Modalidad taller sobre Evaluación de la innovación de la docencia.

${ }^{* *}$ Magister en Educación - CINDE. Intereses de investigación: Educación y pedagogía, gestión y evaluación de proyectos sociales y educativos, metodologías de investigación de corte cualitativo y procesos de incorporación de TIC en educación. Calle 18A No. 0 - 19 Este. Casita Rosada 2o. piso. Bogotá, Colombia. Universidad de los Andes, Colombia. Profesora de planta del Centro de Investigación y Formación en Educación (CIFE). 571-3394949 ext 4806. E-mail: $<$ maldana@uniandes.edu.co $>$.

${ }^{* * *}$ Administrador de la Universidad de los Andes (Bogotá, Colombia). Actualmente es Asistente Graduado de tiempo completo en la Facultad de Administración de la misma universidad, donde se desempeña como docente e investigador. Es coautor de los libros "Lo social y lo económico: ¿Dos caras de una misma moneda?” con José Camilo Dávila, Carlos Dávila y Lina Grisales (2011) y "Marketing para emprendedores" (2010) con Alejandro Schnarch. Sus intereses en investigación son: capacidades organizacionales y perdurabilidad; consultoría como herramienta de administración; además se interesa por metodología de aprendizaje.

${ }^{1}$ Esta sección ha tomado apartes del programa del curso "Casos y Proyectos en Administración" (Uniandes, 2011).

${ }^{2}$ En 2002 la Facultad fue acreditada por EQUIS de la "European Foundation for Management Development"; en 2003 recibió la acreditación AMBA de la "Association of MBAs"; y en 2010 obtuvo la acreditación de AACSB, otorgada por la "Association to Advance Collegiate Schools of Business".

${ }^{3}$ Puede entenderse como curso integrador, aquel propio de "una experiencia de culminación en la que los estudiantes deben aplicar las habilidades y conocimientos adquiridos a lo largo del programa" (PALOMBA; BANTA, 2001).

${ }^{4}$ Centro de investigación y formación en educación.

${ }^{5}$ Elaborada de manera colaborativa por Álvaro Triana y César Cadavid, profesores de cátedra del curso durante el primer semestre de 2011.

${ }^{6}$ Elaborado por Montoya, J. En presentación sobre "Diseño integrado de cursos". CIFE - Universidad de los Andes, (2010).

7 "Saber declarativo o proposicional se refiere al saber sobre las cosas o 'saber qué."' (BIGGS, 1999, p. 63).

8 "El conocimiento funcional se basa en la idea de actuaciones fundamentadas en la comprensión. Estos conocimientos están en la experiencia del aprendiz." (BIGGS, 1999, p. 63).

${ }^{9}$ Así se evidenció en algunas respuestas de los estudiantes en la evaluación del curso en 2009-2. 